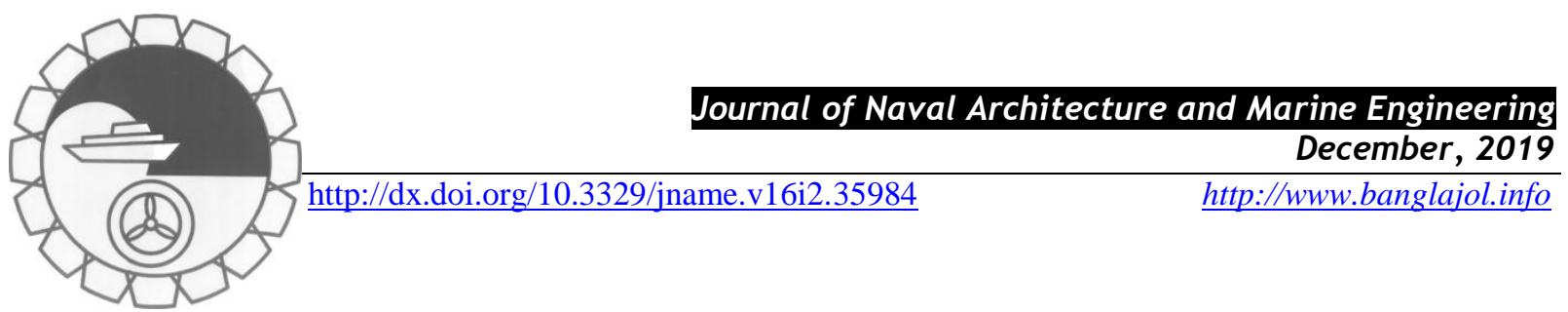

\title{
A PANEL METHOD FOR BOTH MARINE PROPULSION AND RENEWABLE ENERGY
}

\author{
Y. Xu ${ }^{1,3}$, P. Liu ${ }^{2 *}$, I. Penesis ${ }^{1}$ and G. He ${ }^{4}$ \\ ${ }^{1}$ Australian Maritime College, University of Tasmania, Tasmania 7250, Australia \\ $2^{2 *}$ Marine Technology, School of Engineering, Newcastle University, Newcastle upon Tyne, NE1 7RU, UK, \\ Email: pengfei.liu@ncl.ac.uk \\ ${ }^{3}$ Guangxi University of Science and Technology, Liuzhou, 545006 China \\ ${ }^{4}$ International School of Ocean Science and Engineering, Harbin Institute of Technology, Weihai, 264200, China
}

\begin{abstract}
:
A computational hydrodynamics method was formulated and implemented as a tool from screw propeller propulsion to renewable energy performance prediction, design and optimization of horizontal axis turbines. As an example, for tidal energy generation, a comparative analysis between screw propellers and horizontal axis turbines was presented, in terms of geometry and motion parameters, inflow velocity analysis and the implementation methodologies. Comparison and analysis are given for a marine propeller model and a horizontal axis turbine model that have experimental measurements available in literature. Analysis and comparison are presented in terms of thrust coefficients, shaft torque/power coefficients, blade surface pressure distributions, and downstream velocity profiles. The effect of number of blades from 2 to 5, of a tidal turbine on hydrodynamic efficiency is also obtained and presented. The key implementation techniques and methodologies are provided in detail for this panel method as a prediction tool for horizontal axis turbines. While the method has been proven to be accurate and robust for many propellers tested in the past, this numerical tool was also validated and presented for both tidal and wind turbines.
\end{abstract}

Keywords: Panel method; marine propeller; renewable energy turbine, aero-dynamics, hydrodynamics etc.

\section{Introduction}

Computational methods have been widely used for wind and tidal turbine research and development. A comprehensive review of these methods and their merits and limitations, for example, was given by NichollsLee et al. (2008). Among these methods, the panel methods, in the most advanced and complicated method group, have both high computing efficiency and prediction accuracy as an engineering tool for turbine simulation and design optimization. While probably the blade element methods (BEM) are the most widely used as preliminary simulation tools for wind and tidal turbines, much fewer penal methods could be found for turbine in literature. To the author's knowledge, these few panel methods for turbine simulation and prediction include, a 2D panel method by Drela et al. mentioned in (Nicholas-Lee et al., 2008), a 3D time domain panel method for wind turbine by Hampsey (2002), a rudder-propeller interaction panel code by Turnock (2007) and a design-based simulation work by Greco et al. (2007). Formulation and implementation of these panel methods for both wind turbines and tidal turbines are basically the same though different fluid properties such as viscosity/density and hence Reynolds number, require different schemes and numerical treatments. The primary difference for panel methods as tools for wind turbine and for tidal turbine is that tidal turbines may be exposed to cavitation that would cut off the negative pressure spike and reduces the energy extraction efficiency substantially even if there does not exist stall or separation. For wind turbine under high speed inflow conditions, compressibility might not be a problem, at least in terms of turbine efficiency correction. A bare panel method developed from scratch also needs many other essential numerical components for different application cases. Establishing these numerical components for a newly developed bare panel method requires substantial effort and implementation development, and long term and substantial effort in validation, as described later in this section. In this paper, it shows that a well-established panel method can be used for both turbine simulation and propulsion.

Panel methods are also called the boundary element methods, or boundary integral methods (BEM in short as well). Lifting surface and panel methods have been widely used in research and development of aircraft wings, hydrofoils and both aerial and marine propellers. Zero thickness propeller blade simulated and computed by 
lifting surface theory in the computational fluid dynamics (CFD) field has a history of over 60 years. The use of the surface panel method for a simple body surface mesh can be traced back to the early 1960s. Hess and Vararezo (1985) probably made the first panel method computation for propellers. To deal with complete aircraft geometry, panel method codes, PMARC (Panel Method Ames Research Center) developed by Katz (Ashby et al., 1991) and VSAERO by Maskew (1986) are the early examples of panel methods for aircraft wings and propellers. On the other hand, panel methods have also been used for marine propeller research development and early examples among those are publications by Kerwin (Greeley and Kerwin, 1982)] and Hoshino (1989), just to name a few. A time domain unsteady panel method code OSFBEM (oscillating foil boundary element method) was developed by the Liu (1996) for oscillating propulsors of both chordwise and spanwise flexibility to simulate marine animals' propulsion (Liu 1996). To respond the need in simulation of fluid-structure interactive hydrodynamics to predict ice blockage effects between ice sheet and ice-class propellers, a panel method code, Rotorysics (formally PROPELLA, Liu, 1996b) was developed in 1996, based on OSFBEM. Since then, continued efforts were made to maintain and enhance the capability of the panel method. The capability for unsteady oblique flow and inflow wake were presented in early 1998 at 1989 ITTC Propeller RNS/Panel Method Workshop. At the meantime, a pre- and postprocessor was developed for the code by using OpenGL and Visual C++ of Microsoft Foundation Class, as a 3D unsteady data visualization tool to view the geometry motion and color blended results. A novel and robust numerical Kutta condition using Broyden's iteration was developed. Since 2003, this panel method has been redeveloped with a multiple-body interaction formulation to deal with a propeller with pod and strut, called a podded propeller unit and recently for a podded propulsor interacting with an ice body at variable proximity (transient hydrodynamic response of a propeller moving towards an ice sheet). In the past decades, many panel methods have been developed and these panel methods along with their associated numerical schemes and techniques for propellers are well established. These existing panel methods with a minor or moderate revision, could be quickly used for tidal and wind turbine prediction. The aim of this work is to present the physics and numerical similarities and difference between propeller and turbine panel methods and then provide a detailed implementation techniques and treatments, to establish a panel method for turbine aero- and hydrodynamics prediction.

\section{Theoretical Background}

The current panel method is a multiple-body interaction panel method. The fundamentals of panel method have been presented in detail in some textbooks, including the ones by Moran (1984) for 2D foil sections and by Katz and Plotkin (1991) for unsteady 3D body and wings. A detailed formulation and implementation for a loworder, time-domain panel method, were given by Liu (1996).

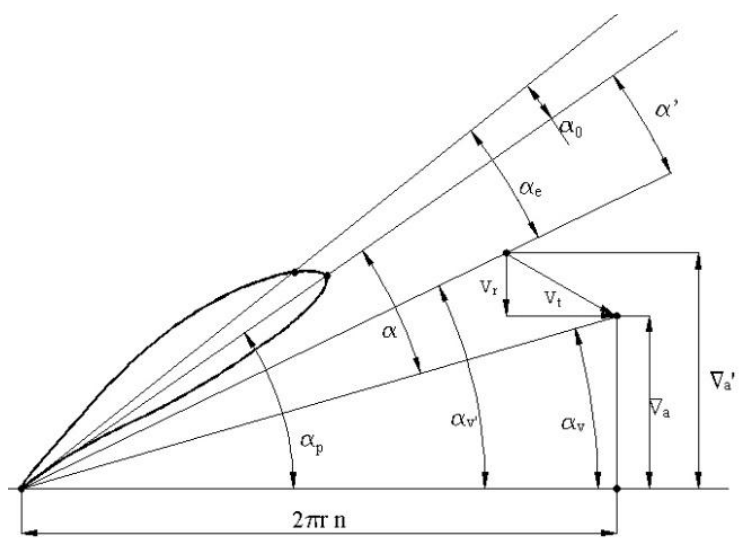

Fig. 1: Propeller blade section velocity schematic diagram.

\subsection{Flow physics similarity and differences between propeller and tidal turbine}

Being rotary wings, flow around both propeller and turbine blade sections has both similarities and differences. Flow conditions around a wing section determine the hydrodynamic characteristics of the wing section. These characteristics are mainly determined by the effective angle of attack of the blade section. The similarity and differences of the flow around a blade section between propeller and turbine are shown in the velocity diagrams in Figs. 1 and 2 respectively. 
In Fig. 1, the blade geometric pitch angle of attack is represented as $\alpha_{p}$, and the angle of zero life of the blade section is $\alpha_{o}$ due to the camber of the blade section. Therefore, the effective geometric angle of pitch is $\alpha_{p}+\alpha_{o}$. The inflow velocity angle of attack, also called hydrodynamic angle of attack in some literature, is $\alpha_{v}=$ $\tan ^{-1}\left(\frac{V_{\alpha}}{2 \pi r n}\right)$, where $V_{a}$ is propeller shaft forward velocity, also called inflow velocity in some literature, and $r$ is the blade section local radius and $n$ is the shaft rotation speed in revolutions per second. However, even in open water, as a propeller is rotating, the inflow velocity to the propeller plane is not unidirectional because of the induced tangential velocity $V_{t}$ and radial velocity $V_{r}$. In fact, these induced velocities are not in the same plane as shown in the figure. The total inflow velocity relative to the local blade section in $3 D$ space is then $\overrightarrow{V_{a}}+\overrightarrow{V_{t}}+$ $\overrightarrow{V_{r}}$ with the resultant velocity angle of attack $\alpha^{\prime}$.

Therefore, the effective angle of attack of the blade section, that determines the hydrodynamic characteristic of a blade section, is $\alpha_{e}=\alpha_{p}+\alpha_{o}-\alpha_{V}^{\prime}$, for which induced velocities have been taken into account, and is $\alpha_{e}=\alpha_{p}-\alpha_{V}$, without including the induced velocities and angle of zero life. It is obvious that for a propeller to produce positive thrust, i.e., to be in propulsion mode, the effective angle of attack must be positive. In the current version of the panel method code Rotorysics, the angle of shed wake vortices at the blade trailing edge is taken as $\alpha$ at $r=0.7000 R$, where $\mathrm{R}$ is the radius of the propeller, when the angle of zero life is neglected. When shed wake vortices roll-up is considered, the angle of shed wake vortices is modified by the induced velocity during a wake vortex relaxation procedure. While the pitch angle of shed wake vortices has substantial effect on the prediction accuracy of thrust and torque prediction, it is even more important to the accuracy of the field velocity prediction downstream of a propeller and hence the minimization of the prediction uncertainty. In addition, to avail a multiple-body computational capability, all bodies in the fluid domain move individually in an acquiescent fluid.

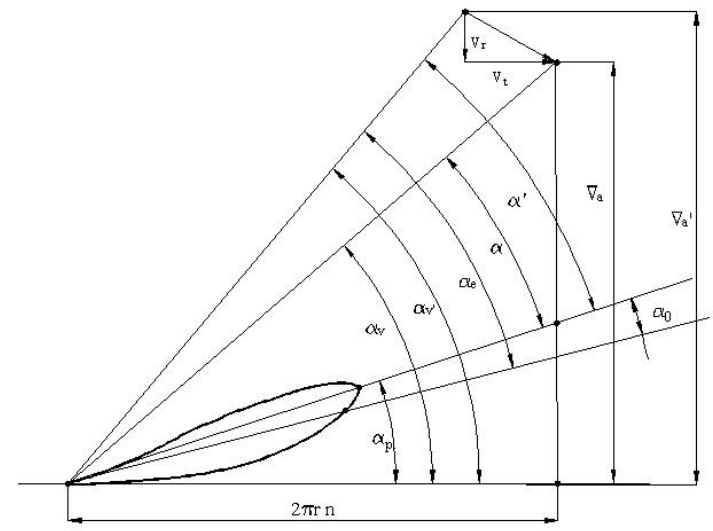

Fig. 2: Turbine blade section velocity schematic diagram.

A similar blade section velocity diagram for turbine is shown in Fig. 2. With all the same variables as those for propeller, the offset coordinates of the surfaces of propeller blade section are now interchanged, i.e., the suction side and pressure side of the blade section are swapped for a turbine. The effective angle of attack now becomes

when the induced velocities are considered, and

$$
\alpha_{e}=\alpha_{V}^{\prime}-\alpha_{p}+\alpha_{o}
$$

$$
\alpha_{e}=\alpha_{V}-\alpha_{p}
$$

when the induced velocities and angle of zero lift are both neglected. It is also obvious that the effective angle of attack must be positive for the blade section to be in turbine mode. Now using the propeller code Rotorysics for turbine prediction is reduced to two key modifications when preparing for input, that is, the blade sectional coordinates interchange and the formulation and implementation of the angle of shed wake vortices. Using the same motion and geometric variables for propellers, the angle of shed wake vortices for a turbine is taken as $\alpha$ at local radius of $0.7 R$. In the following non-dimensional analysis, the wake pitch angle of turbine was obtained as follows:

$$
\begin{aligned}
\alpha & =\tan ^{-1} \frac{V_{a}}{2 \pi r n}-\tan ^{-1} \frac{n p}{2 \pi r n} \\
& =\tan ^{-1} \frac{J D n}{2 \pi r n}-\tan ^{-1} \frac{\frac{p}{D} D}{2 \pi r} \\
& =\tan ^{-1} \frac{J}{\pi \frac{r}{R}}-\tan ^{-1} \frac{p_{D}}{\pi \frac{r}{R}},
\end{aligned}
$$


where $J$ is advance coefficient, $J=\frac{V_{a}}{n D}$, of turbine and $P_{D}$ is the local non-dimensional pitch ratio, $p_{D}=\frac{p}{D}$, based on the diameter of turbine. The same as for propeller simulation, turbine is moving in an acquiescent fluid; the pitch ratio at $70 \%$ radius is taken for wake vortices formulation, as $p_{W D}=J-\left.P_{D}\right|_{0.7 R}$. The angle of shed wake vortices for a propeller by using the similar formulation gives good thrust and torque prediction and induced velocity estimation downstream. However, for turbine mode after extensive numerical test runs, the angle of shed wake vortices with an additional angle of inflow taken as $p_{W D}=2 J-\left.P_{D}\right|_{0.7 R}$ gave better results. Hoshino (1989) recommended a wake vortex discretization formulation based on LDV measurements downstream of a propeller, including wake pitch, wake contraction, ultimate wake region, and transition. This formulation is valid only for propulsion mode, not turbine mode. For turbine, if this wake vortex discretization is used, wake contraction becomes a substantial inflation (800 times), which should be prohibited and avoided. This occurred at a low tip speed ratio of about $T S R=4.0000$ corresponding to $J=0.7855$, with a ratio of $\frac{J}{p_{D}}$ at about 3 , in the tidal turbine example (see the results and discussion section below).

Fig. 3 shows the discretized wake vortices behind a 3-blade P4119 propeller (Jessup, 1989) at $J=0.8330$ and a $20^{\circ}$ root pitch tidal turbine at $T S R=7.0000(\mathrm{~J}=0.4488)$. In this paper, the tidal turbine refers to the tidal turbine model by Bahaj et al (2007). For presentation purposes, wake vortices in Fig. 3 are shown only for one blade.

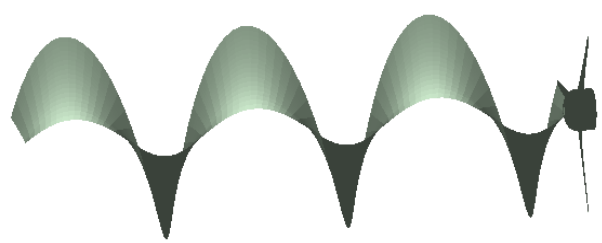

Turbine wake vortices

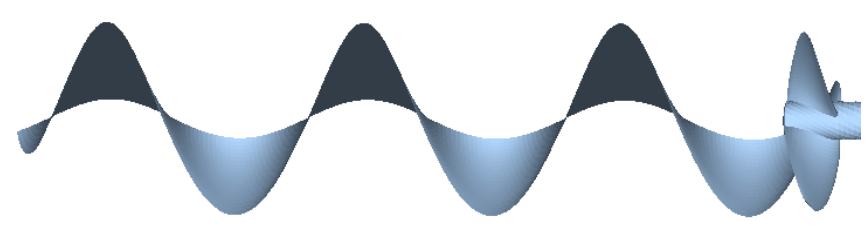

Propeller wake vortices

Fig. 3: Shed wake vortices discretization for the DTMB propeller P4119 and the 20_root pitch tidal turbine.

\subsection{Flow physics similarity and differences between propeller and tidal turbine}

To predict renewable energy performance and perform a coupled design and optimization of both propeller and turbine, a numerical tool for both propellers and turbines was capability-enhanced based on the in-house code Rotorysics recently (Liu et al., 2018).

Two major numerical capability enhancements were completed for the simulation tool Rotorysics. They are A) the vortex-shedding model that involves in the formulation of the pitch and stretch of the shed-vortices immediate after the trailing edge, especially for turbine mode. Validation of this numerical enhancement took substantial time and effort in computation; B) the innovative formulation and implementation of all 4 quadrants of operation and the establishment of the automation to switch between operation quadrants. A traditional panel method can only handle the first quadrant operation.

\subsection{Four Quadrant Simulation for Turbine and Propeller}

Forward and reverse shaft revolution speeds and inflow speeds produces four operation quadrants for a propeller/turbine. Fig. 4 shows the Rotorysics definition of the 4-quadrant operating conditions of a propellerrotor. The ordinate is revolution speed and is positive upward; and the abscissa is inflow speed and positive when it comes to the propeller-rotor. 


$$
\begin{array}{c|c}
\text { Q2: } V_{\text {prop }} \leq 0, n>0 & \begin{array}{r}
\text { Q1: } V_{\text {prop }} \geq 0, n>0 \\
V_{\text {prop }}
\end{array} \\
\hline \text { Q3: } V_{\text {prop }} \leq 0, n<0 & \text { Q4: } V_{\text {prop }} \geq 0, n<0
\end{array}
$$

Fig. 4: Definition of propeller/turbine operating in four quadrants. $V_{\text {prop }} \geq 0$ when blade section upper side facing inflow; shaft speed $\mathrm{n}>0$ (right hand rotation viewing from stern to bow) when the LE facing the inflow.

Definition of propeller/turbine operating in four quadrants. $V_{\text {prop }} \geq 0$ when blade section upper side facing inflow; shaft speed $n>0$ when the LE facing the inflow.

Definition of propeller/turbine operating in four quadrants. $V_{\text {prop }} \geq 0$ when blade section upper side facing inflow; shaft speed $n>0$ when the LE facing the inflow.

The 4 quadrants defined in Fig. 4 can also be divided in to two modes, propulsion mode and turbine mode. Below is a summary of the 4 quadrant operations:

In the 1st quadrant, $V_{\text {prop }} \geq 0, n>0$ and this is a normal forward propulsion mode;

In the 2nd quadrant, $V_{\text {prop }} \leq 0, n>0$ and this is a normal turbine power generation mode;

In the 3rd quadrant, $V_{\text {prop }} \leq 0, n<0$ and this is a reserve/astern propulsion mode;

In the 4th quadrant, $V_{\text {prop }} \geq 0, n<0$ and this is a reverse inflow turbine power generation mode.

\subsection{Panel method to simulate 4-quadrant operations}

A panel method requires the shed vortices to always go downstream. In Rotorysics both the inflow and shaft revolution directions are pre-set and remain the same, i.e., propeller-rotor moves in the negative $X$ direction in the global coordinates and its shaft rotates clockwise viewed in the direction from stern to bow. Quadrant 1 with both $V_{\text {prop }}$ and $n$ positive, is the default. To simulate an operation in other quadrants, by just reversing either shaft speed $n$ or inflow $V_{\text {prop }}$ will create inverse vortices in the reverse flow case or opposite vortex shedding edge from the L.E in the reverse revolution case, which will violate panel method theoretical establishment and will produce incorrect results, because there will be interaction between shed vortices and surface panels creating numerical singularities. The reversion of inflow speed or switching the revolution direction could be programmed but a simpler approach with much less programming effort was done for Rotorysics as the follows: With the shaft revolution direction remaining the same, swapping only U.S. to L.S. of the blade sections, swapping both U.S. to L.S. and the L.E. to T.E., or swapping only the L.E. to T.E., will create a propeller-rotor operating conditions in quadrants 2, 3 and 4, respectively. The details are shown in Fig. 5. Motion parameters $J$ for propulsion, TSR (converted to $J$ ) for turbine and $n$ in the input for Rotorysics are always positive. The predicted $K_{t}$ and $K_{q}$ for propulsion at the same time are converted to $C_{t}$ and $C_{\text {pow }}$ for turbine. When $J=0$, propulsion efficiency $\eta=0$, turbine tip speed ratio $T S R=\infty, C_{t}=\infty$ and $C_{\text {pow }}=\infty$ by definitions.

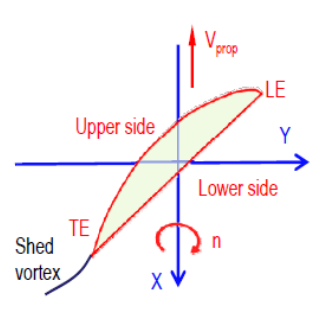

(a) Q1

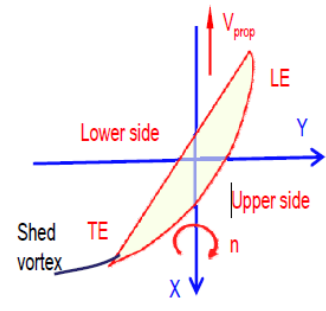

(b) Q2

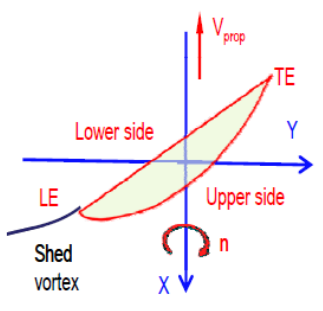

(c) Q3

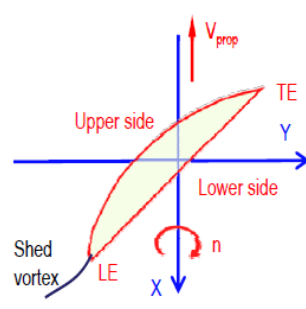

(d) Q4

Fig. 5: Rotorysics to simulate 4-quadrant operations for propulsion mode, turbine mode, astern propulsion mode and reverse inflow turbine mode. 
When the U.S. of blade sections facing inflow, the value of $V_{\text {prop }}$ is negative (propeller-rotor moving forward); when L.S facing inflow, the values of $V_{\text {prop }}$ in real flow condition is positive (propeller-rotor moving astern); When the L.E. facing inflow, the values of $n$ in real operating condition, is positive (right hand rotation) and when the T.E. facing inflow, the value of $n$ in real operating condition, is negative (left hand rotation).

In the reverse inflow turbine mode in quadrant 4 , the rotor is experiencing a reverse wind/tide and hence the inflow facing the U.S. of the blade section, i.e., $V_{\text {prop }}$ pointing at upward on the page so inflow comes towards the U.S.

\subsection{The flow velocity and effective angle of attack (AoA) of a rotor}

\subsubsection{Effective AoA of a Propeller Blade Section in the 1st Quadrant}

An effective angle of attack concept was introduced to analyze the instantaneous flow condition for an unsteady 3D foil with both pitch and heave along with translation motion (Liu, 1996). The same idea is used here to analyze the flow condition, namely the load/force of a propeller-rotor blade section. In Fig. 6, blade geometric pitch angle of attack is represented by $\alpha_{p}$, and the angle of zero life of the blade section is $\alpha_{0}$ due to the camber of the blade section. Therefore, the effective geometric angle of pitch is $\alpha_{p}+\alpha_{0}$. The inflow velocity angle of attack, also called hydrodynamic angle of attack in the literature, is $\alpha_{v}=\tan ^{-1}\left(\frac{V_{a}}{2 \pi r n}\right)$, where $V_{a}$ is inflow speed (opposite to the motion of the propeller rotor $V_{a}=-V_{\text {prop }}$ ), and $r$ is the blade section local radius and $n$ is the shaft rotation speed in revolutions per second. However, even in open water, as a propeller is rotating, the inflow velocity to the propeller plane is not unidirectional because of the induced tangential velocity $V_{t}$ and radial velocity $V_{r}$. In fact, these induced velocities are not in the same plane as shown in the figure. The total inflow velocity relative to the local blade section in $3 \mathrm{D}$ space is then $\overrightarrow{V_{a}}+\overrightarrow{V_{t}}+\overrightarrow{V_{r}}$ with the resultant velocity angle of attack $\alpha_{v}{ }^{\prime}$. Therefore, the effective angle of attack of the blade section, that determines the performance characteristics (lift, drag, etc.) of a blade section, is $\alpha_{e}=\alpha_{p}-\alpha_{0}-\alpha_{v}{ }^{\prime}$ for which induced velocities have been taken into account and is $\alpha=\alpha_{p}-\alpha_{v}$ without including the induced velocities and angle of zero lift.

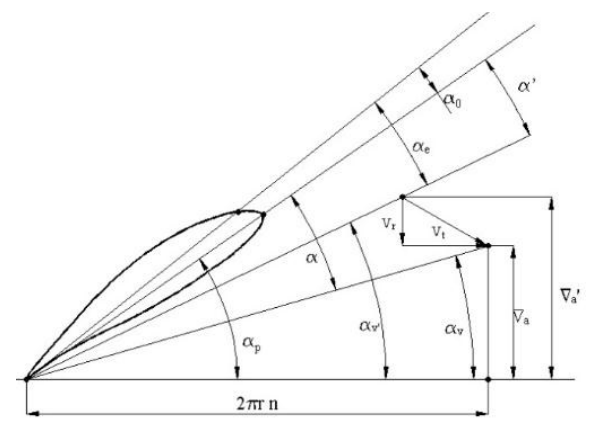

Fig. 6: Effective AoA of a propeller blade section (Liu, 2010).

It is obvious that for a propeller to produce thrust, i.e., in the 1st or the 3rd quadrants, the effective angle of attack must be positive. In Rotorysics the angle of shed wake vortices at the blade trailing edge is taken as $\alpha$ at $r=0.70 R$, where $R$ is the radius of the propeller, when the angle of zero life is neglected. When the roll-up of the shed wake vortices is considered, the angle of shed wake vortices is modified by the induced velocity during a wake vortex relaxation procedure. While the pitch angle of shed wake vortices has substantial effect on the prediction accuracy of thrust and torque prediction, it is even more important for the accuracy of the field velocity prediction downstream of a propeller. Rotorysics was programmed to handle multiple-body simulation simultaneously and all bodies in the fluid domain move individually in an acquiescent fluid.

The following is a summary of the flow situation around a propeller blade section defined by Rotorysics in the 1st quadrant:

- The upper side of the propeller blade section faces the inflow;

- In Rotorysics, the rotor always moves in an acquiescent fluid so that this arrangement allows multiplebody interaction (rotor, rudder, nozzle, ice blockage, etc.) and rotates in the right hand direction;

- The higher the pitch of the rotor, the higher the effective AoA; 
- The propeller is usually behind the stern of a ship hull so the whole inflow due to hull shape usually has substantial influence on propeller performance (Rotorysics takes inflow wake field input, if needed).

- The thrust and torque created in the 1 st and 3rd quadrant in practical propulsion applications are usually very large and so is the consumed power.

\subsubsection{Effective AoA of a turbine blade section in the 2nd quadrant}

A similar blade section velocity diagram for turbine is shown in Fig. 7. With all the same variables as those for propeller, the offset coordinates of the surfaces of the propeller blade section are now interchanged, i.e., the suction and pressure side of the blade section are swapped to construct for a turbine flow. The effective angle of attack now becomes $\alpha_{e}=\alpha_{v}{ }^{\prime}-\alpha_{p}+\alpha_{0}$ when the induced velocities are considered and $\alpha=\alpha_{v}-\alpha_{p}$ when the induced velocities and angle of zero lift are both neglected.

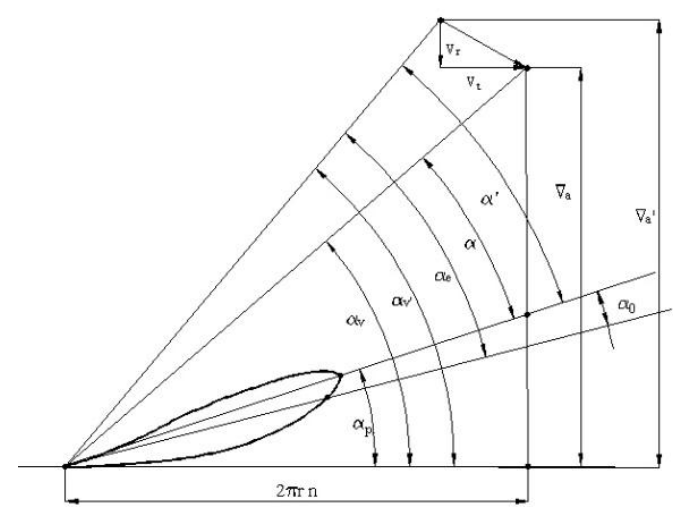

Fig. 7: Effective AoA of a turbine blade section (Liu, 2010).

The following is a summary of the flow situation around a turbine blade in the 2nd quadrant:

- The L.S. of the turbine rotor section faces inflow;

- In Rotorysics rotor always moves in an acquiescent fluid and rotates in the right hand direction;

- Contrary to propulsion mode, the higher the pitch of the turbine blade section, the smaller the effective AoA;

- To reduce the cut-off speed of the turbine rotor, i.e. for the rotor to start to spin at low inflow speed (equivalent to a very large tip speed ration TSR), or to maximize power generation $P$ for a small inflow speed $V_{a}$, the pitch $p_{D}$ should be reduced to a very small value to provide a sufficiently large effective angle of attack;

- For power generation efficiency, too small a value of $p_{D}$, close to or less than zero, will produce a large effective AoA but this should be avoid because torque (i.e. power) created by the lift projected in the direction of the torque vector will be zero or negative and therefore the generated power would be close zero or negative (Liu, 2010);

- Torque and drag on turbine rotors are much smaller than that on propeller (about or less than 1/10) of the same diameter. Therefore, the blade sectional thickness could be designed much smaller than the propeller to save blade material;

- For most ocean tidal turbines, cavitation number is big $(\mathrm{Cn}=80$ was found for a 20 -m turbine (Liu and Bose, 2012)) because turbine rotors have a much lower shaft speed and much larger operating depth. For deep ocean tidal turbines, therefore, cavitation is not a concern.

\subsubsection{Parametric Interpolation between propeller and tidal turbine}

The geometric parameters between a propeller blade and a tidal turbine are the same. For example, the expanded area ratio EAR is the ratio of the total blade area to the area of the propeller disk. Therefore, the solidity of a turbine rotor is equivalent to the EAR of a propeller. For a tidal turbine calculation using a propeller code such 
as Rotorysics, the only geometry manipulation needed to prepare for code input if the source code is for propeller mode, is to interchange the blade upper side with the lower side, i.e. to swap the suction side and the pressure side; this could also be done with the same geometry input for propeller but the swap is performed in the source code. However, to extrapolate the results in propeller format corresponding to a turbine convention, three major variables need to be interpolated. They are advance coefficient $\mathrm{J}$ versus tip speed ratio TSR, propeller thrust coefficient $\mathrm{K}_{T}$ versus turbine thrust coefficient $\mathrm{C}_{T}$, and propeller torque coefficient $\mathrm{K}_{Q}$ versus power coefficient $\mathrm{C}_{\text {pow }}$. With the definitions of propeller advance coefficient, $J=\frac{V_{a}}{n D}$, propeller thrust coefficient $K_{T}=\frac{T_{\text {thrust }}}{\rho n^{2} D^{4}}$, propeller shaft torque coefficient $K_{Q}=\frac{Q_{\text {torque }}}{\rho n^{2} D^{5}}$, tip speed ratio $T S R=\frac{\omega R}{V_{a}}$, and turbine thrust coefficient, $C_{T}=\frac{T_{\text {thrust }}}{\frac{1}{2} \rho V_{a}^{3} A}$, the three parameters for turbine in terms of propeller, can be derived as the following:

and

$$
\begin{gathered}
T S R=\frac{\omega R}{V_{a}}=\frac{2 \pi n R}{V_{a}}=\frac{\pi D}{V_{a}}=\frac{\pi}{J}, \\
C_{T}=\frac{T_{\text {thrust }}}{\frac{1}{2} \rho V_{a}^{2} A}=\frac{K_{T} \rho n^{2} D^{4}}{\frac{1}{2} \rho V_{a}^{2} \pi R^{2}}=\frac{8 K_{T}}{\pi J^{2}},
\end{gathered}
$$

$$
C_{\text {pow }}=\frac{Q_{\text {torque }} \omega}{\frac{1}{2} \rho V_{a}^{3} A}=\frac{K_{Q} \rho n^{2} D^{5} 2 \pi n R}{\frac{1}{2} \rho V_{a}^{3} \pi R^{2}}=\frac{16 K_{Q} n^{3} D^{3}}{V_{a}^{3}}=\frac{16 K_{Q}}{J^{3}} .
$$

Therefore, for a desired TSR a value of corresponding advance coefficient $\mathbf{J}$ can be obtained for a propeller code. When the results $\mathrm{K}_{T}$ and $\mathrm{K}_{Q}$ are obtained from a propeller code, they can be interpolated as $\mathrm{C}_{T}$ and $\mathrm{C}_{\text {pow }}$, respectively, for turbine as a function of non-dimensional speed $\mathrm{J}$.

\section{Results and discussion}

\subsection{Geometry and motion parameters}

Predictions for turbine hydrodynamic characteristics were obtained for a tidal turbine base model plus two root pitch offsets. For these models, experimental measurements are available for comparison. The turbine model section was taken as NACA 63-8xx section (Abbot and von Doenhoff, 1949). The turbine blade's sectional maximum thickness varies from $24.0000 \%$ at the root section to $15.6000 \%$ at the tip section.

A model propeller P4119 by David Taylor Model Basin (DTMB) along with its results is used for comparison (Jessup, 1989). Surface panel mesh arrangements for the propeller, the tidal turbine models studied in (Bahaj et al., 2007) and the solid modeling of the turbine rotor optimized by Liu and Bose (2012) for an energy site in the Bay of Fundy, are shown in Fig. 8.

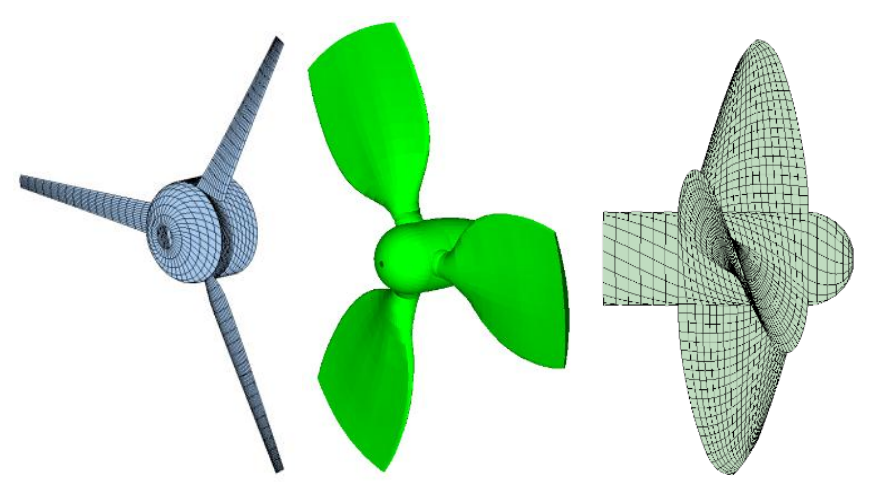

Fig. 8: Surface mesh and geometry shape comparison among the DTMB propeller P4119, the optimized rotor geometry for the Bay of Fundy (Liu and Bose 2012) and the 20-degree root pitch tidal turbine (Bahaj et al., 2007). 
To obtain a range of tip speed ratio TSR from 4.0000 to 10.0000 , corresponding input values of $\mathrm{J}$ and required shaft revolution speed $\mathrm{N}$ in rpm for a 0.8000 -m diameter tidal turbine model were obtained. These $\mathrm{J}$ values were calculated based on a constant inflow speed of $1.5000 \mathrm{~m} / \mathrm{s}$ to simulate the actual cavitation tunnel test conditions by Bahaj et al. (2007) and are listed in Table 1.

Table 1: Input turbine motion parameters in terms of $\mathrm{J}$ and $\mathrm{N}$

$\begin{array}{llll}\text { TSR } & \mathrm{J} & \mathrm{n}=\mathrm{Va} / \mathrm{J} / \mathrm{D} & \mathrm{N}(\mathrm{rpm}) \\ 4.0000 & 0.7854 & 2.3873 & 143.2394 \\ 4.5000 & 0.6981 & 2.6857 & 161.1444 \\ 5.0000 & 0.6283 & 2.9842 & 179.0493 \\ 5.5000 & 0.5712 & 3.2826 & 196.9542 \\ 6.0000 & 0.5236 & 3.5810 & 214.8592 \\ 6.5000 & 0.4833 & 3.8794 & 232.7641 \\ 7.0000 & 0.4488 & 4.1778 & 250.6690 \\ 7.5000 & 0.4189 & 4.4762 & 268.5740 \\ 8.0000 & 0.3927 & 4.7746 & 286.4789 \\ 9.0000 & 0.3491 & 5.3715 & 322.2888 \\ 10.0000 & 0.3142 & 5.9683 & 358.0986\end{array}$

Table 2 shows the sectional effective angle of attack (AoA) at shaft speed of $n=4.0000$ rps corresponding to a TSR of 6.7021. The values of the effective angle of attack are estimated when the induced velocity and angle of zero lift are neglected. In the work by Bahaj et al. (2007), a base turbine model has a root pitch angle of $15^{\circ}$ with an offset degree of $5^{\circ}$ and $10^{\circ}$ to add another two more turbine models.

Table 2: AoA of the 0.8000-meter diameter turbine blade sections with a shaft speed of $n=4.0000$, inflow velocity $\mathrm{V}_{a}=1.5000 \mathrm{~m} / \mathrm{s}, \mathrm{TSR}=6.7021$ and corresponding $\mathrm{J}=0.4688$ (induced velocities and angle of zero lift were neglected).

\begin{tabular}{|c|l|l|l|l|l|l|}
\hline $\mathrm{r} / \mathrm{R}$ & \multicolumn{1}{|c|}{$\mathrm{r}$} & $\mathrm{V}_{\text {resutant }}$ & $\tan ^{-1} \frac{V_{a}}{\omega r}$ & $\alpha=15^{\circ}$ pitch & $\alpha=20^{\circ}$ pitch & $\begin{array}{c}\alpha=25^{\circ} \\
\text { pitch }\end{array}$ \\
\hline 0.2000 & 2.0106 & 2.5085 & 36.7244 & 21.7244 & 16.7244 & 11.7244 \\
\hline 0.2500 & 2.5133 & 2.9269 & 30.8301 & 18.7301 & 13.7301 & 8.7301 \\
\hline 0.3000 & 3.0159 & 3.3684 & 26.4439 & 16.9439 & 11.9439 & 6.9439 \\
\hline 0.3500 & 3.5186 & 3.8250 & 23.0889 & 15.4889 & 10.4889 & 5.4889 \\
\hline 0.4000 & 4.0212 & 4.2919 & 20.4565 & 14.3565 & 9.3565 & 4.3565 \\
\hline 0.4500 & 4.5239 & 4.7661 & 18.3441 & 13.4441 & 8.4441 & 3.4441 \\
\hline 0.5000 & 5.0265 & 5.2456 & 16.6159 & 12.7159 & 7.7159 & 2.7159 \\
\hline 0.5500 & 5.5292 & 5.7291 & 15.1783 & 12.0783 & 7.0783 & 2.0783 \\
\hline 0.6000 & 6.0319 & 6.2156 & 13.9650 & 11.5650 & 6.5650 & 1.5650 \\
\hline
\end{tabular}




\begin{tabular}{|l|l|l|l|l|l|l|}
\hline 0.6500 & 6.5345 & 6.7045 & 12.9283 & 11.0283 & 6.0283 & 1.0283 \\
\hline 0.7000 & 7.0372 & 7.1953 & 12.0327 & 10.5327 & 5.5327 & 0.5327 \\
\hline 0.7500 & 7.5398 & 7.6876 & 11.2517 & 10.0517 & 5.0517 & 0.0517 \\
\hline 0.8000 & 8.0425 & 8.1812 & 10.5648 & 9.6648 & 4.6648 & -0.3352 \\
\hline 0.8500 & 8.5451 & 8.6758 & 9.9562 & 9.3562 & 4.3562 & -0.6438 \\
\hline 0.9000 & 9.0478 & 9.1713 & 9.4132 & 9.0132 & 4.0132 & -0.9868 \\
\hline 0.9500 & 9.5504 & 9.6675 & 8.9260 & 8.7260 & 3.7260 & -1.2740 \\
\hline 1.0000 & 10.0531 & 10.1644 & 8.4864 & 8.4864 & 3.4864 & -1.5136 \\
\hline
\end{tabular}

Examining the sectional effective AoA for the $15^{\circ}, 20^{\circ}$ and $25^{\circ}$ root pitch angle turbines, the $15^{\circ}$ root pitch turbine is the most heavily loaded. The effective AoA at the tip section is about $8.50^{\circ}$, which will most likely result in a bad cavitation (cavitation analysis is a different topic so it is omitted here though Rotorysics has the capability to do so). The best sectional effective AoA among these three turbines is the $20^{\circ}$ root pitch one, though a slight increase of effective AoA up to $5^{\circ}$ at the tip might give a better hydrodynamics efficiency. The $25^{\circ}$ root pitch turbine would give the poorest hydrodynamic efficiency because the blade sections from $\mathrm{r} / \mathrm{R}=$ 0.8000 to the tip are all in propulsion mode (the effective AoA is negative by turbine AoA definition).

\subsection{Turbine $\mathrm{C}_{\boldsymbol{T}}$ and $\mathrm{C}_{\text {pow }}$ versus propeller $\mathrm{K}_{\boldsymbol{T}}$ and $\mathrm{K}_{\boldsymbol{Q}}$}

Figs. 9, 10 and 11 show the thrust and power coefficient of the horizontal axis turbine with a root pitch angle of $15^{\circ}, 20^{\circ}$ and $25^{\circ}$.

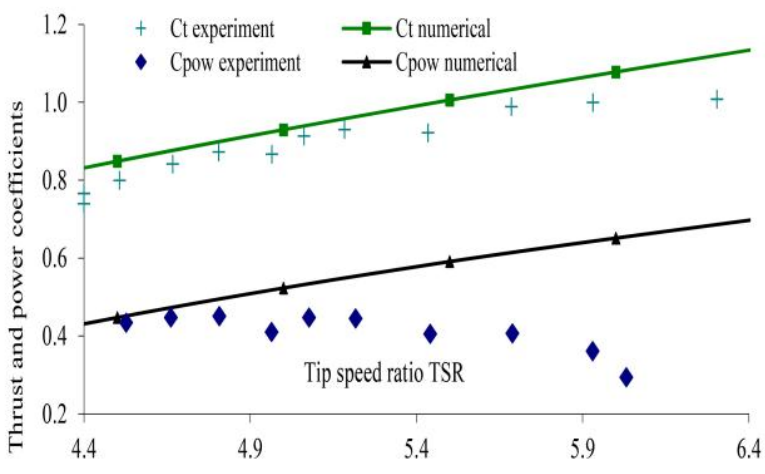

Fig. 9: Comparison of thrust coefficient $\mathrm{C}_{\mathrm{T}}$ and power coefficient $\mathrm{C}_{\text {pow }}$ predicted by Rotorysics with the measurements (Bahaj et al., 2007) for the base turbine model of $15^{\circ}$ root pitch angle (zero degree of offset).

As mentioned earlier in this section, the effective angles of attack of the blade section of the $15^{\circ}$ root pitch turbine are abnormally large. These large values could most likely cause flow separation and stall. The separation and stall simulation are not implemented in the current method. It is noted too that the maximum power coefficient of the $15^{\circ}$ root pitch turbine is just as high as about 0.4000 and this value diminishes at a TSR of about 6.0000. This small production of power coefficient are most likely attributed separation and stall where potential flow-based methods like the panel methods could not simulate (the higher the TSR, the worse the stall and separation and hence the larger the discrepancy between the method and measurements). This indicates that the $15^{\circ}$ root pitch angle turbine is operational only in a narrow range of tip speed ratio with poor hydrodynamic efficiency. 


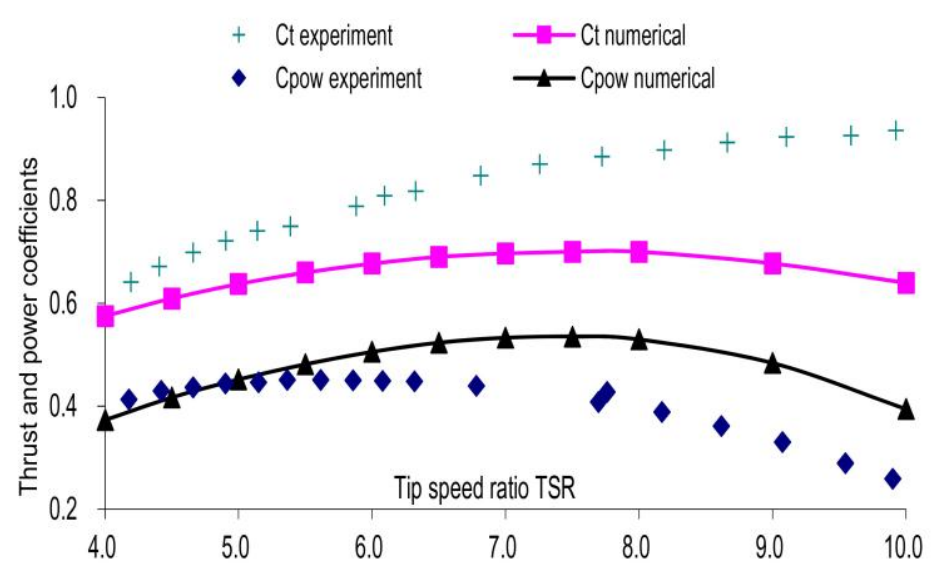

Fig. 10: Comparison of thrust coefficient $\mathrm{C}_{\mathrm{T}}$ and power coefficient $\mathrm{C}_{\text {pow }}$ predicted by Rotorysics with the measurements (Bahaj et al., 2007) for the turbine model of $20^{\circ}$ root pitch angle (5-degree offset).

In Fig. 10, a better hydrodynamic performance is shown for the $20^{\circ}$ root pitch angle turbine. The current method produced a slightly lower thrust coefficient and higher power coefficient, compared with the measurements, than that of the $15^{\circ}$ root pitch turbine, especially operating under at large tip speed ratio close to 10.0000 . It noted that the maximum power coefficient occurred at the tip speed ratio TSR of 6.0000 for measurements but occurred at about a TSR of 7.5000 for the prediction.

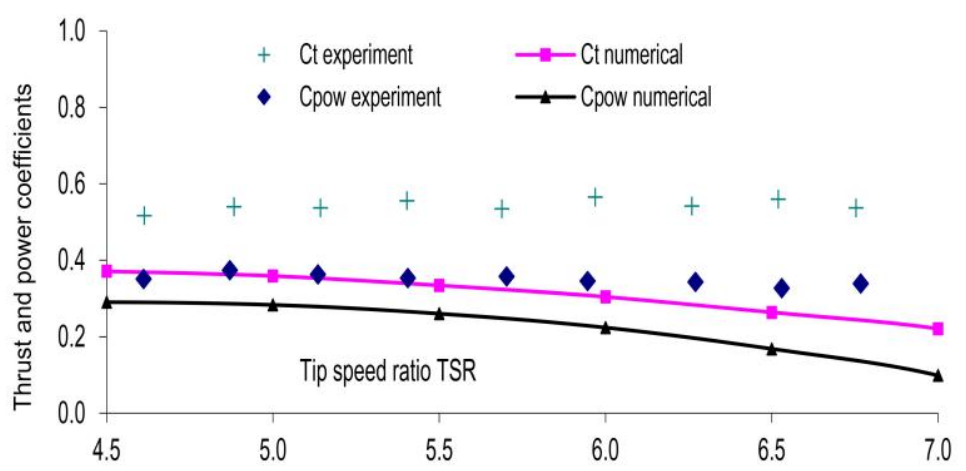

Fig. 11: Comparison of thrust coefficient $C_{T}$ and power coefficient $C_{\text {pow }}$ predicted by Rotorysics with the measurements (Bahaj et al., 2007) for the turbine model of $25^{\circ}$ root pitch angle (10-degree offset).

A general agreement between the measurements and the prediction by the current method can also be seen for the $25^{\circ}$ root pitch angle turbine. As mentioned earlier in this section, blade tip sections at more than $80 \% R$ radial locations have negative effective angle of attack and hence they do negative work. This caused a much small power coefficient compared with the $20^{\circ}$ one $(0.3900$ versus 0.4500$)$.

As mentioned earlier for wake vortices discretization, the wake pitch angle has a strong influence on the prediction accuracy of the method. Taking a proper value of wake pitch will ensure not only the accuracy of predicted thrust and power coefficients but also for the velocity profile downstream. At the moment, lack of sufficient tidal turbine measurements on thrust and power coefficients, and experimental data by LDV or PIV for the velocity profile downstream of a tidal turbine, makes it difficult for numerical method validation.

Fig. 12 shows a set of thrust and power coefficient for the DTMB propeller by the current method Rotorysics. 


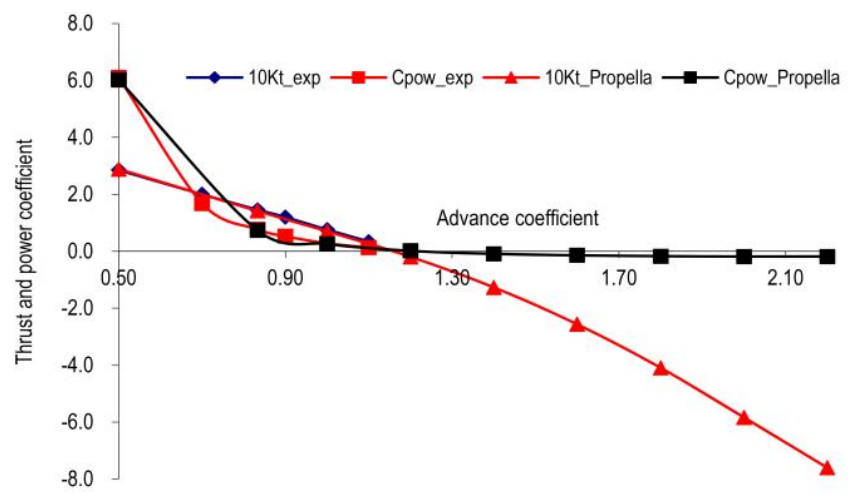

Fig. 12: Comparison of predicted thrust coefficient $\mathrm{K}_{T}$ and power coefficient $\mathrm{C}_{\text {pow }}$ with the measurements for the DTMB P4119 propeller (Jessup, 1989).

Prediction by Rotorysics agreed very well with the measurements. As described above, the wake pitch was taken as the value of the angle of attack at $0.7000 \mathrm{R}$ and this wake pitch seems a proper one based on the close agreement between the prediction and the measurements. Further comparison will be given for the induced velocity at a location downstream of propeller and turbine.

\subsection{Pressure distribution of a propeller versus a tidal turbine}

Fig. 13 shows the pressure distribution on the blade surface of the DTMB P4119 propeller at an advance coefficient of $\mathrm{J}=0.8330$, compared with that of the tidal turbine. The values of the pressure coefficient on the back side of the blade (viewing from downstream) are mainly negative. Therefore, the back side of a propeller blade is often referred to suction side and the face side is referred to the pressure side, when operating in the propulsion mode at the first quadrant (positive rotational speed and positive forward speed). After a robust numerical Kutta condition was applied, the pressure difference between the suction side and the pressure side at the trailing edge, as can be seen in the figure, is close to zero. The pressure distribution for the tidal turbine is shown for the pressure side, which is also on the same side of the propeller viewing from the downstream.

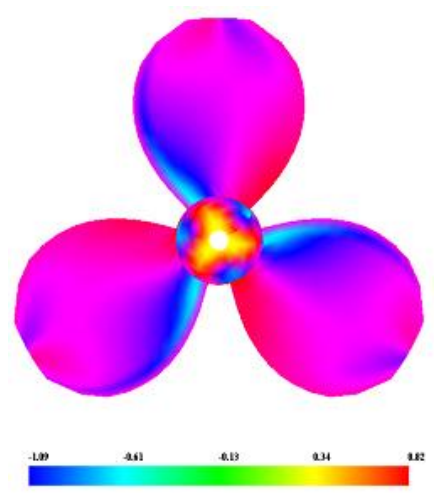

Fig. 13: Color blended pressure distribution on the DTMB P4119 propeller blades.

Fig. 14 shows the pressure distribution on a blade of the $20^{\circ}$ root pitch tidal turbine at $T S R=7.0000$. It is noted that, contrary to propeller, the values of the pressure coefficients on the back side of the tidal turbine (viewing from downstream) are mainly positive and these on face side are mainly negative. The back side now becomes pressure side and vice versa. It also can be seen that the values of the suction side pressure of the tidal turbine could possibly cause severe cavitation that would deteriorate the hydrodynamic performance substantially, if the shaft immersion depth of tidal turbine is sufficiently small. 

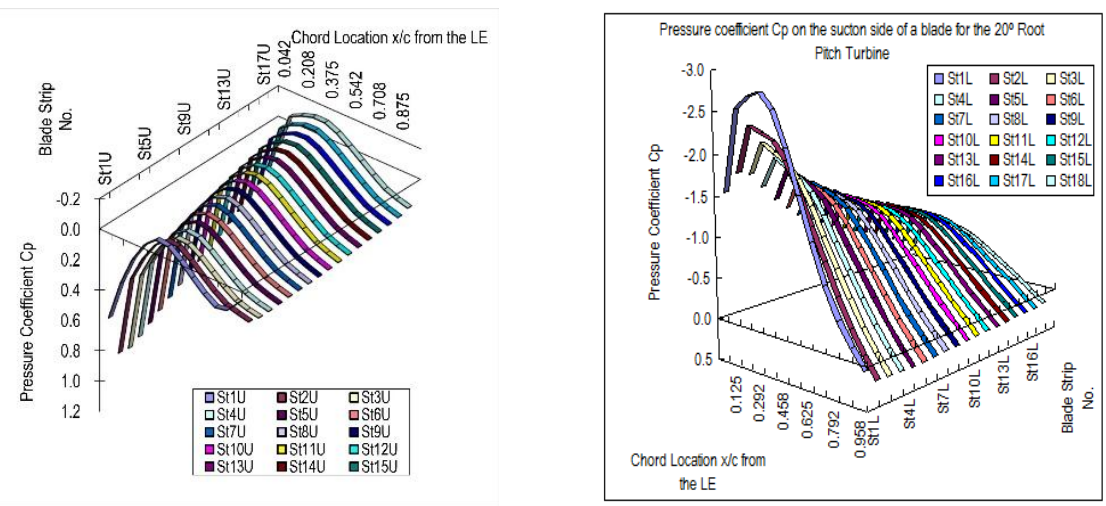

Fig. 14: Unfolded blade section pressure distribution of the $20^{\circ}$ root pitch tidal turbine at TS R $=7: 0000$.

Fig. 15 shows the color blended pressure coefficient distribution on the surface of the right-handed rotation tidal turbine blades, viewing from downstream. High pressure occurs at the trailing edge of the blade sections and the pressure coefficient decreases with the chord location towards the leading edge.

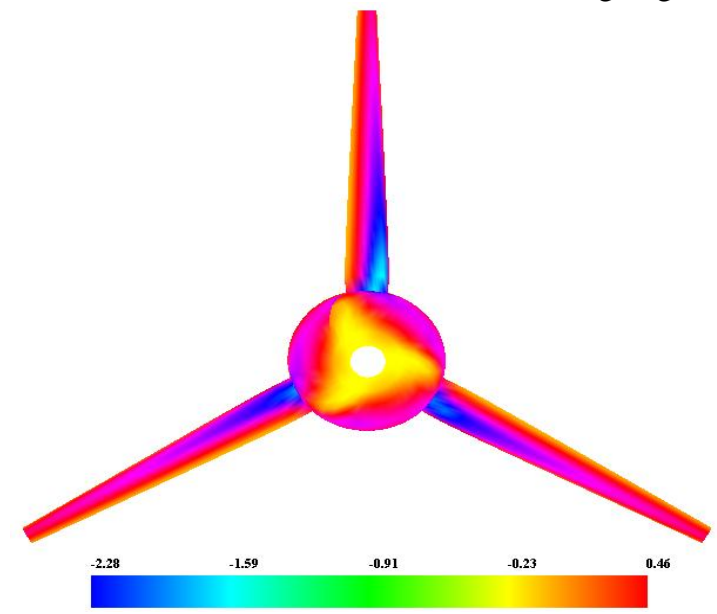

Fig. 15: Blended color presentation of the pressure coefficient distribution on the surface of the $20^{\circ}$ root pitch tidal turbine.

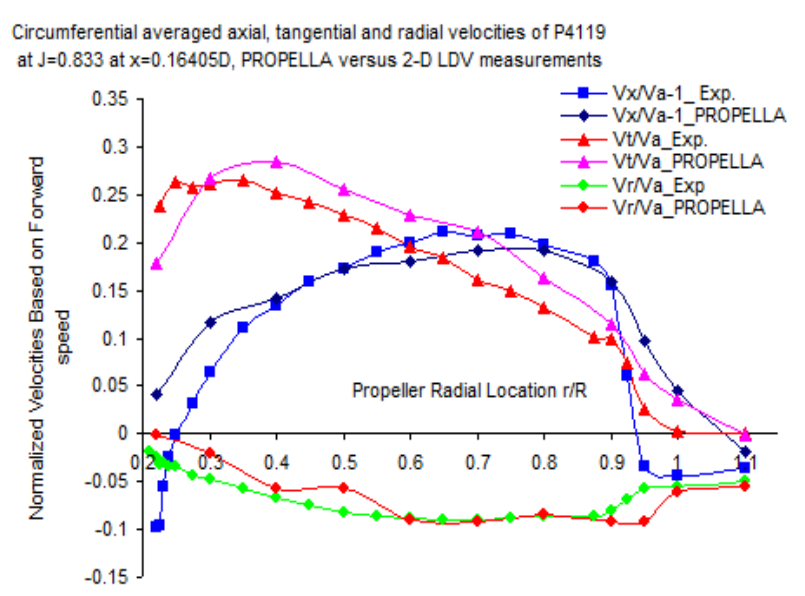

Fig. 16: Velocity profile at a disk plane of 0.16405 times of the diameter of the DTMB P4119 propeller downstream at $\mathrm{J}=0.8330$, prediction versus experiment (Jessup, 1989). 


\subsection{Downstream velocity profile: propeller versus tidal turbine}

A comparison on velocity profile downstream of the propeller and the tidal turbine is discussed below. Fig. 16 shows the velocity profile in a plane at 0.16405 diameter behind the propeller predicted by the current panel method Rotorysics compared with the measurement by using LDV (Jessup, 1989). It is noted that the tangential velocity is in the same direction with the revolution direction of the propeller shaft. The axial velocity at the plane pointing downstream but radial velocity is negative, parallel to centre-line of the shaft. The negative radial velocity means that the shed wake vortices at this plane are in contraction.

Fig. 17 presents the predicted velocity profile at a plane of 0.16405 diameter downstream of the tidal turbine at $T S R=7.0000$. Contrary to propeller, the radial velocity at the plane is positive which means that the wake vortices tend to inflate. The tangential velocity is opposite to the rotation of the turbine with negative sign except the tip. The axial velocity is negative as well indicating that the flow past the blades of the turbine has a decrease in velocity.

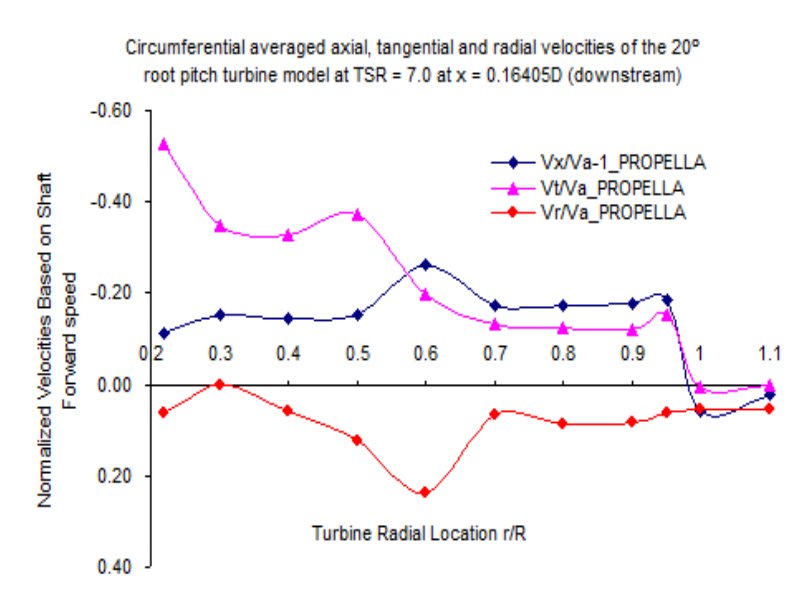

Fig. 17: Velocity profile at a disk plane of 0.16405 times of the diameter of the $20^{\circ}$ root pitch tidal turbine at TSR $=7.0000$.

The opposing direction of the downstream velocities of a propeller against that of a turbine also indicates that propeller in operation transfer energy to the fluid to accelerate the inflow, while turbine extracts energy by absorbing the momentum of the fluid and ends up a slowing down the inflow.

\subsection{Number of blade effect on hydrodynamic efficiency of tidal turbines}

A numerical investigation was also made to examine the effect of number of blades on the hydrodynamic efficiency (power coefficient) for a turbine of 2,3,4 and 5 blades with the same solidity, i.e., expanded area ratio $E A R=0.0669$. Fig. 18 shows the surface solid modeling of the turbine geometry for the 4 turbines of different number of blades.
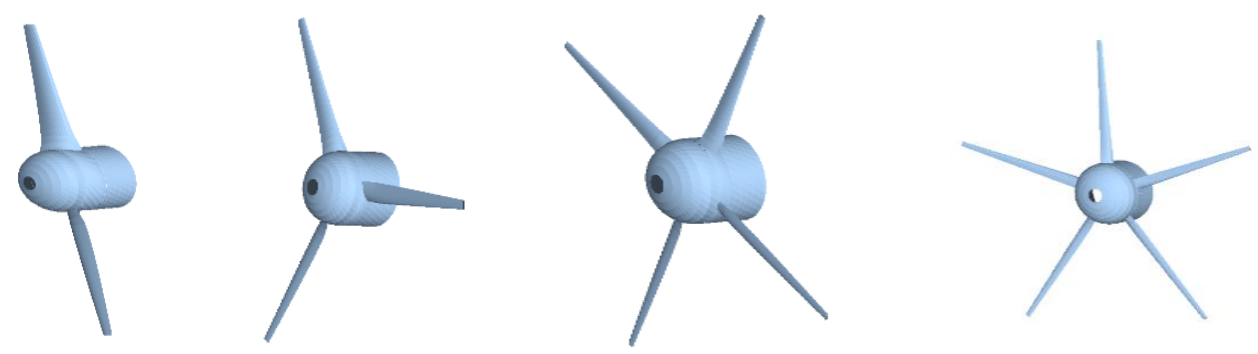

Fig. 18: Solid surface modeling of the 4 tidal turbines of different number of blades.

Fig. 19 presents a comparison of the hydrodynamic efficiency (power coefficients) among the 2-, 3-, 4- and 5blade tidal turbines. The geometry of the 4 tidal turbines was generated by a constant expanded area ratio the same as the 3-blade tidal turbine model. The chord length of the turbine blades was enlarged proportionally for 
the 2-blade turbine and reduced for the 4- and 5-blade turbines. As it shows in Fig. 19, with the increase of the number of blades, power coefficients dropped gradually and proportionally. With the increase of the number of blades, the TSR values at which the maximum power coefficients TSR occur, shift to the left (reduced), from $T S R=8.0000$ for the 2-blade tidal turbine to $T S R=6.5000$ for the 5-blade one.

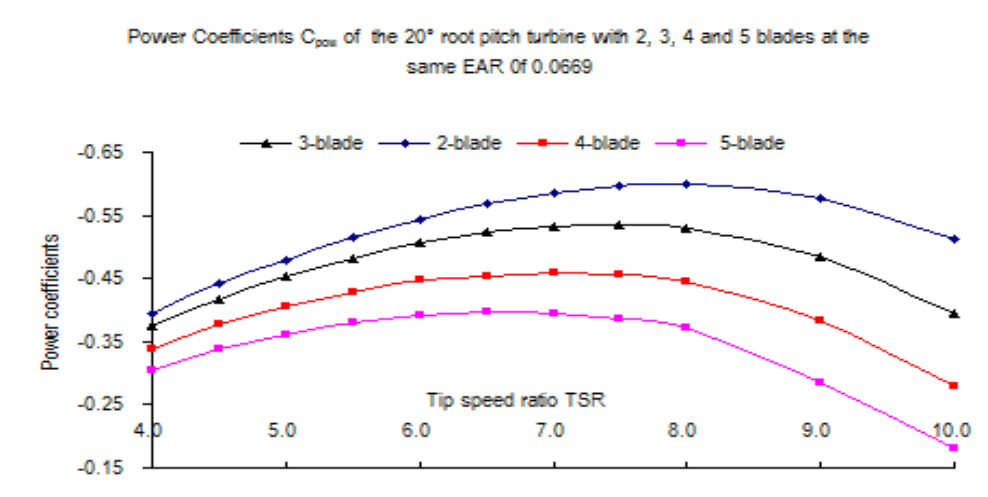

Fig. 19: Power coefficients of 2-, 3-, 4- and 5-blade tidal turbines of $20^{\circ}$ root pitch angle.

\subsection{Discussion on source of certainties}

There are the following sources of uncertainties:

- The largest uncertainity for panel methods is the shed wake vortices alignment. Shed vortices alignment of this panel method was validated against experimental data after a substantial validation and trails runs (over 10,000 of runs).

- The second largest source of error is the time step size of the panel method code. The time step size determination for accuracy and reliability of the panel method is a traditional problem that has been resolved at the beginning stage of the code development in the 1990's.

- The least source of error is the programming precision. The hydrodynamic kernel of this panel method is written in ANSI C with double precision (16-digits accuracy). The input geometry of the foil section for the rotor sections is only single precision as ANSI $\mathrm{C}$ reading function can only read a single precision float number. At the initial development stage, it was proved that the accuracy of geometry by single precision has little effect on the overall precision.

\section{Conclusions}

A time-domain, low order panel method was developed for tidal turbine performance evaluation, design and optimization, based on a well-established and robust propeller code Rotorysics. Similarities and differences in hydrodynamic characteristic between propeller and turbine were discussed in terms of blade section flow velocity diagram and effective angle of attack. Detailed methodology and techniques were presented for propeller panel method applied to migrate to simulation for turbines. Predictions for a propeller and a turbine model were obtained and compared. These predictions showed a general agreement with the measurements. Blade surface pressure distributions and the downstream velocity profile of the tidal turbine model obtained are contrary to a propeller. A numerical investigation was performed for 4 turbines with the same geometry shape and same expanded area ratio, EAR, with a different number of blades ranging from 2 to 5 . Results indicate that with the increase of the number of blades, the power coefficients decrease dramatically and proportionally. It was also noted that the optimum TSR at which the highest power coefficient occurs shifts left (reduces) with the increase of the number of blades, from TSR $=8.0000$ to 6.5000 . While the propeller panel method was developed with a huge amount of measurement data to validate it, there is a need for substantial experimental data on wake vortices and downstream velocity profile of turbine rotors by LDV and/or SPIV.

\section{References}

Abbot, I., von Doenhoff, A. (1949): Theory of Wing Sections, Dover Publications, Inc.

Ashby, D. L., Dudley, M., Iguchi, S., Browne, L., Katz, J. (1991): Potential flow theory and operation guide for the panel code PMARC, Tech. Rep. TM 102851, NASA. 
Bahaj, A., Batten, W., McCann, G. (2007): Power and thrust measurement of marine current turbines under various hydrodynamic flow conditions in a cavitation tunnel and a towing tank, Renewable Energy, Vol. 32, No. 3, pp. 407-426. https://dx.doi.org/10.1016/j.renene.2006.01.012

DTI (2007): Economic viability of a simple tidal stream energy device, Tech. Rep. Contract No.TP/3/ERG/6/1/15527/REP, UK Department of Trade and Industry.

Greco, L., Testa, C., Slavato, F. (2007): Design oriented aerodynamic modeling of wind turbine performance, Journal of Physics: Conference Series, Vol. 75, pp. 1-13. https://dx.doi.org/10.1088/1742-6596/75/1/012011

Greeley, D., Kerwin, J. (1982): Numerical methods for propellers design and analysis in steady flow, Transactions of the Society of Naval Architects and Marine Engineers, Vol. 90, pp. 415-453.

Hampsey, M., 2002. Multiobjective evolutionary optimization of small wind turbine blades, PhD thesis, University of Newcastle, Australia.

Hess, J. L., Vararezo, W. (1985): Calculation of steady flow about propellers by mean of surface panel method, Tech. Rep. Proceedings of Research and Technology, Douglas Aircraft Company Long Beach, CA.

https://doi.org/10.2514/6.1985-283

Hoshino, T., 1989. Flow field around propeller (2nd report), Tech. Rep. Society of Naval Architects of Japan.

Jessup, J., 1989. An experimental investigation of viscous aspects of propeller blade flow, PhD thesis, The Catholic University of America.

Katz, J., Plotkin, A. (1991): Low-Speed Aerodynamic-From Wing Theory to Panel Methods, McGraw-Hill, Inc., New York.

Liu, P. (1996b): Software development on propeller geometry input processing and panel method predictions of propulsive performance of the R-Class propeller, Tech. Rep. Report No. 1, MMC Engineering \& Research, TDC Contract No. T8200-6-6507-001/XSD, Newfoundland, Canada.

Liu, P. (1996): A time-domain panel method for oscillating propulsors with both spanwise and chordwise flexibility. PhD thesis, Memorial University of Newfoundland.

Liu, P. (2010): A computational hydrodynamics method for horizontal axis turbine - panel method modelling migration from propeller to turbine, Energy, Vol. 35, No. 7, pp. 2843-285.

https://dx.doi.org/10.1016/j.energy.2010.03.013

Liu, P., Bose, N. (2012): Prototyping a series of bi-directional horizontal axis tidal turbines for optimum energy conversion. Applied Energy, Vol. 99, pp. 50-66. https://dx.doi.org/10.1016/j.apenergy.2012.04.042

Liu, P., Bose, N., Chen, K., Xu, Y. (2018): Development and optimization of dual-mode propellers for renewable energy. Renewable Energy, Vol. 119, pp. 566-576. https://dx.doi.org/10.1016/j.renene.2017.12.041

Maskew, B. (1986): Program VSAERO theory document, Tech. Rep. Contractor Report 4023, NASA.

Moran, J. (1984): Introduction to Theoretical and Computational Aerodynamics, John Wiley and Sons, New York.

Nicholls-Lee, R., Turnock, S., Boyd, S. (2008): Simulation based optimization of marine current turbine blades, 7th International Conference on Computer and IT Applications in the Marine Industries (COMPIT'08), Liege. COMPIT'08, pp. 314-328. 